\title{
COMPLEX STRUCTURES ON RIEMANN SURFACES
}

\author{
BY
}

\section{GARO KIREMIDJIAN}

\begin{abstract}
Let $X$ be a Riemann surface (compact or noncompact) with the property that the length of every closed geodesic is bounded away from zero. Then we show that sufficiently small complex structures on $X$ can be described without making use of Schwarzian derivatives or the theory of quasiconformal mappings. Instead, we use methods developed in Kuranishi's work on the existence of locally complete families of deformations of compact complex manifolds.

We introduce norms ||$_{k}(k$ a positive integer $)$ on the space of $C^{\infty}(0, p)$ forms with values in the tangent bundle on $X$, which are similar to the usual Sobolev \|\|$_{k}$-norms. (In the compact case ||$_{k}$ is equivalent to \|\|$_{k}$.) Then we prove that certain properties of \|\|$_{k}$, crucial for Kuranishi's approach, are also satisfied by ||$_{k}$
\end{abstract}

0. Introduction. The idea of deformation of a Riemann surface was initiated by Riemann who computed the number of parameters on which the deformation depends. In more recent years $O$. Teichmüller based the study of the space of complex structures of a compact Riemann surface on the theory of quasiconformal mappings.

The most remarkable progress in this direction was made by L. Ahlfors and L. Bers whorigorously developed the ideas initiated by Teichmüller and showed that the space of classes of equivalent Riemann surfaces has a complex structure (see [1], [4]). Moreover, their work extended the theory to the case of open

Riemann surfaces where it was established that the space of classes of equivalent complex structures on an arbitrary Riemann surface is isomorphic to a bounded domain in a complex Banach space ([2], [5]).

In $1957 \mathrm{~K}$. Kodaira and D. Spencer [6] made the first steps toward developing a systematic theory of deformations of compact higher dimensional manifolds.

They defined the notion of a universal family of deformations of a compact complex manifold and gave examples of manifolds for which such families exist. The general problem of existence of universal families was solved by M. Kuranishi ([7], [8]).

The purpose of this paper is to describe sufficiently small complex structures on Riemann surfaces without making use of quasiconformal mappings and Schwarzian

Received by the editors April 5, 1971 and, in revised form, September 17, 1971. AMS 1970 subject classifications. Primary 32G05, 32G15; Secondary 30A52.

Key words and phrases. Complex manifold, Riemann surface, almost complex and complex structures, deformations of complex structures, holomorphic vector bundles, Laplace operator, $W^{D, q}$-ellipticity, Green's operator, $k$-norms. 
derivatives. At the same time it is illustrated how Kuranishi's methods can be extended to certain noncompact manifolds.

In $\$ 1$ we list certain differential operators on complex manifolds and give the ir explicit expressions for Riemann surfaces. We also give a proof of the simple but important fact that the tangent bundle of every nonexceptional Riemann surface is $W^{0,0}$-elliptic (see also [3]).

$\S 2$ deals with standard facts about almost complex and complex structures which are taken from [9].

In $\$ 3$ we proceed toward proving the main theorem (Theorem 3.10). First, on the space of $(0,0)$ and $(0,1) C^{\infty}$ forms with values in the tangent bundle of the Riemann surface $X$ we introduce the norm ||$_{k}$. One of the important properties of this norm (and also a crucial fact in our argument) is given by Proposition 3.4: $|G \phi|_{k+2} \leq c_{k}|\phi|_{k}$ for sections $\phi$ of the tangent bundle. This is Friedrichs' inequality with respect to ||$_{k}$. We also need a Sobolev inequality with respect to ||$_{k}$ (Proposition 3.5.1 and Corollary 3.5.2). In order to obtain it, we impose a certain geometric condition on $X$. More specifically, we require that there exists a number $t>0$ such that the length of every closed geodesic is greater than or equal to $t$. We note that this property holds for compact Riemann surfaces and also for a large class of noncompact Riemann surfaces. We should point out that this condition is imposed by the approach we are taking. As it was said before, the result we are after is known for arbitrary Riemann surfaces using the theory of quasiconformal mappings and Schwarzian derivatives.

Now we have the tools to apply the implicit function theory for Banach spaces in order to solve differential equation $\delta\left(\omega(s) \circ f^{s}\right)=0$ for diffeomorphisms $f^{s}: X \rightarrow X$. This enables us to complete the proof of our

Main Theorem. The universal family for complex structures, represented by $(0,1) C^{\infty}$ forms with finite ||$_{k}$-norm is the set $B_{k}$ of $C^{\infty}(0,1)$ forms $\omega$ of the form $\omega=\lambda^{-2} \bar{\psi}$, with $|\omega|_{k}<\infty$ and $\sup _{X}|\omega|<1$, where $\lambda$ is the complete Poincaré metric on $X$ and $\psi$ is a bolomorphic quadratic differential.

I would like to extend my gratitude to Professors Lipman Bers and Masatake Kuranishi for their valuable advice during the preparation of this paper.

1. Preliminaries. Let $X$ be a complex manifold and let $E \stackrel{\pi}{\rightarrow} X$ be a holomorphic vector bundle over $X$ with fiber $C^{m}$. Let $\mathcal{U}=\left\{U_{i}\right\}_{i \in I}$ be a coordinate covering of $X$ such that $E / U_{i}$ is isomorphic to $U_{i} \times C^{m}$. Let $e_{i j}: U_{i} \cap U_{j} \rightarrow \mathrm{GL}(m, C)$ be the holomorphic cocycle defined by the conditions that $\Phi_{i}\left(z, \xi_{i}\right)=\Phi_{j}\left(z, e_{i j} \xi_{j}\right)$ where $\Phi_{i}$ are the isomorphisms $U_{i} \times C^{m} \rightarrow E$ and $\xi_{i}$ are the fiber coordinates over $U_{i}$. The dual bundle $E^{*} \rightarrow X$ is defined on the same covering $\mathcal{U}$ by ${ }^{t} e_{i j}^{-1}$.

For example, the tangent bundle $\theta$ will be defined in terms of a choice of local coordinates $\left(z_{i}^{\prime}, \cdots, z_{i}^{n}\right)$ on $U_{i}$ by the cocycle $J_{i j}=\partial\left(z_{i}\right) / \partial\left(z_{j}\right)$. 
1. 1. Definition. $A C^{\infty}$ form of type $(p, q)$ with values in $E$ is a $C^{\infty}$ section of the bundle $E \otimes \theta^{* p} \otimes \bar{\theta}^{* q}$, where $\theta^{* p}=\Lambda^{p} \theta^{*}$ and $\bar{\theta}^{*}$ denotes the complex conjugate of $\theta^{*}$.

Thus, locally on $U_{i}$, such a form will be given by a column vector

$$
\phi_{i}=\left[\begin{array}{c}
\phi_{i}^{\prime} \\
\vdots \\
\phi_{i}^{m}
\end{array}\right]
$$

such that, on $U_{i}, \phi_{i}^{j}$ are $C^{\infty}$ forms of type $(p, q)$ and, on $U_{i} \cap U_{j}, \phi_{i}=e_{i j} \phi_{j}$. The vector space of $C^{\infty}$ forms of type $(p, q)$ with values in $E$ will be denoted by $C^{p, q}(X, E)$.

Next, we introduce a hermitian metric $d s^{2}=2 \Sigma g_{\alpha \bar{\beta}} d z^{\alpha} d \bar{z}^{\beta}$ on $X$ and a hermitian metric on the fibers of $E$. The latter will be given by a hermitian scalar product $b(u, v), u, v \in \pi^{-1}(z)$, which depends differentiably (i.e. $C^{\infty}$ ) on the base point $z$. Locally on $U_{i}$, if $\xi_{i}$ and $\eta_{i}$ are the fiber coordinates of $u$ and $v$, $b(u, v)={ }^{t} \bar{\eta}_{i} b_{i} \xi_{i}$, where $b_{i}$ is a positive definite hermitian matrix in particular, a hermitian metric on the fibers of the holomorphic tangent bundle $\theta$ will be the datum on $X$ of the hermitian metric $d s^{2}$.

1. 2. Definition. We introduce the following operators on the space $C=$ $\bigoplus C^{p, q}(X, E)$ :

$$
\bar{\partial}: C^{p, q}(X, E) \rightarrow C^{p, q+1}(X, E),
$$

the exterior differentiation with respect to the complex conjugates of the local holomorphic coordinates: It has the following property: $\partial \delta=0$.

$$
\text { * : } C^{p, q}(X, E) \rightarrow C^{n-q, n-p}(X, E) \quad\left(n=\operatorname{dim}_{C} X\right),
$$

locally defined by

$$
* \phi=c \operatorname{det}\left(g_{\alpha, \bar{\beta}}\right) \sum \operatorname{sgn}\left(K^{c} K\right) \operatorname{sgn}\left(L^{c} L\right) \phi^{\overline{L^{c}} K^{c}} d z^{K} \wedge d \bar{z}^{L} .
$$

We explain the notation. First of all, $\phi=\Sigma \phi_{I J}-d z^{I} \wedge d \bar{z}^{J}$ where $I=\left(i_{1}, \ldots, i_{p}\right)$, $J=\left(j_{1}, \cdots, j_{q}\right), i_{1}<\cdots<i_{p}, j_{1}<\cdots<j_{q}, 1 \leq i_{\alpha} \leq n, 1 \leq j_{\beta} \leq n$, and $d z^{I}=$ $d z^{i} \wedge \ldots \wedge d z^{i p}, d \bar{z}^{J}=d \bar{z}^{j} \wedge \ldots \wedge d \bar{z}^{j q}$. Further, $K^{c}$ is the increasingly ordered $q$-tuple consisting of the subset of $\{1, \ldots, n\}$ complementary to $K$; and similarly for $L^{c}, \phi^{\bar{L}^{c} K^{c}}=g^{\left(\bar{L}^{c} K^{c}\right)(I \bar{J})} \phi_{I \bar{J}}$, where, if $A$ and $I$ are $p$-tuples and $B$ and $J$ are $q$-tuples,

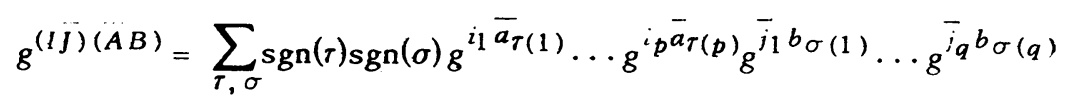

where $\tau$ and $\sigma$ run over all permutations of the sets $\{1, \ldots, p\}$ and $\{1, \ldots, q\}$, respectively. Finally, $c$ is a constant, chosen so that $* * \phi=(-1)^{p+q} \phi$.

$$
\#: C^{p, q}(X, E) \rightarrow C^{q, p}\left(X, E^{*}\right),
$$


which is defined locally, on $U_{i}$, by

$$
(\# \phi)_{i}=\bar{b}_{i} \bar{\phi}_{i}
$$

We note that \# commutes with the operator $*$.

defined by

$$
\delta: C^{p ; q}(X, E) \rightarrow C^{p, q-1}(X, E),
$$

$$
\delta=-\#^{-1} * \bar{\partial} * \# .
$$

We call $\delta$ the formal adjoint of $\bar{\partial}$.

If $\phi, \psi \in C^{p, q}(X, E)^{t} \phi \wedge \# * \psi$ is a scalar $(n, n)$-form which we denote by $A(\phi, \psi) d X, d X$ being the volume element in the considered metric on $X$. In the space $L^{p, q}(X, E)=\left\{\phi \in C^{p, q}(X, E) \mid \int_{X} A(\phi, \phi) d X<\infty\right\}$, the scalar product $(\phi, \psi)=$ $\int_{X} A(\phi, \psi) d X$ is defined and gives $L^{p, q}(X, E)$ the structure of a complex prehilbert space. If supp $\phi \cap \operatorname{supp} \psi$ is compact then $(\vec{\partial} \phi, \psi)=(\phi, \delta \psi)$, for $\phi \in C^{p, q}(X, E), \psi \in C^{p, q+1}(X, E)$. This follows easily by Stokes' theorem.

Let $\mathfrak{D}^{p, q}(X, E)$ be the space of $C^{\infty}$ forms of type $(p, q)$ with values in $E$ with compact support. Let $\mathscr{L}^{p, q}(X, E)$ be the completion of $\mathscr{D}^{p, q}(X, E)$ with respect to the norm $\|\phi\|=(\phi, \phi)^{1 / 2} ; W^{p, q}(X, E)$, the completion of $\mathscr{D}^{p, q}(X, E)$ with respect to the norm $N(\phi)=[(\phi, \phi)+(\bar{\partial} \phi, \bar{\partial} \phi)+(\delta \phi, \delta \phi)]^{1 / 2}$. $W^{p, q}$ is canonically regarded as a vector subspace of $\mathcal{Q}^{p, q}$.

1.3. Definition (see [3]). We say that the vector bundle $E$ is $W^{p, q}$-elliptic if there exists a constant $c>0$ such that, for every $\phi \in \mathfrak{D}^{p, q}(X, E),\|\phi\|^{2} \leq c\left(\|\bar{\partial} \phi\|^{2}+\|\delta \phi\|^{2}\right)$ with respect to some hermitian metric on $X$ and a hermitian metric on the fibers of $E$.

Let $\square=\bar{\partial} \delta+\delta \bar{\partial}: C^{p, q}(X, E) \rightarrow C^{p, q}(X, E)$ be the Laplace-Beltrami operator. W-ellipticity implies the existence of the Green's operator. More precisely, following [3], we have

Theorem 1.4. If the vector bundle $E$ is $W^{p, q}$-elliptic, then, for any $\alpha \epsilon$ $\mathfrak{L}^{p}, q(X, E)$, the equation $\square x=\alpha$ bas one and only one weak solution $x \in W^{p, q}(X, E)$, i.e. for any $u \in W^{p, q}(X, E)$ one has $(\bar{\partial} x, \bar{\partial} u)+(\delta x, \delta u)=(\alpha, u)$. Moreover, if we denote $x$ by $G a$, then there exists a constant $c_{0}>0$ such that

$$
\|G \alpha\|^{2}+\|\bar{\partial} G \alpha\|^{2}+\|\delta G \alpha\|^{2} \leq c_{0}\|\alpha\|^{2} .
$$

Proof. Since $E$ is $W^{p, q}$-elliptic, the form $m(\phi, \psi)=(\bar{\partial} \phi, \bar{\partial} \psi)+(\delta \phi, \delta \psi)$ defines a norm $m(\phi)=m(\phi, \phi)^{1 / 2}$ on $W^{p, q}(X, E)$, which is equivalent to the norm $N(\phi)$. Moreover, $(\phi, \psi)$ gives $W^{p, q}(X, E)$ the structure of a Hilbert space. Let $\alpha \in \mathfrak{L}^{p, q}(X, E)$. Then $u \rightarrow(u, \alpha)$ is a bounded linear functional on $W^{p, q}(X, E)$ with respect to $m(\phi)$, because $|(\alpha, u)| \leq\|\alpha\|\|u\| \leq c^{1 / 2}\|\alpha\| m(u)=M m(u)$. By the Riesz representation theorem, there exists a unique $x \in W^{p, q}(X, E)$ such that $m(x, u)=$ $(\alpha, u)$ for all $u \in W^{p, q}(X, E)$. Moreover, we have that $m(x)=\|H\|$, where $\|H\|$ is 
the norm of the linear functional $H(u)=(u, \alpha)$. Thus $m(x) \leq c^{1 / 2}\|\alpha\|$. Since $m(\phi)$ is equivalent to the norm $N(x)$, there is a constant $c_{0}>0$ such that

$$
N^{2}(x)=\|x\|^{2}+\|\overline{\partial x}\|^{2}+\|\delta x\|^{2} \leq c_{0}\|\alpha\|^{2},
$$

i.e. the linear map $G: \mathfrak{L}^{p}, q(X, E) \rightarrow W^{p, q}(X, E), x=G \alpha$, is continuous. Q.E.D.

Remark 1.5. Since $\square$ represents a strongly elliptic system, it follows from the regularization theorem (see [3]) that if $a \in \mathfrak{L}^{p, q}(X, E) \cap C^{p, q}(X, E)$ then $x \in$ $C^{p, q}(X, E)$ and one has $\square x=\alpha$ in the classical sense.

For the remaining part of this section we assume that $X$ is a nonexceptional Riemann surface, i.e. $X=\Delta / \Gamma$, where $\Delta$ is the unit disc in $\mathrm{C}$ and $\Gamma$ is a Fuchsian group. On $X$ there is a complete hermitian metric $d s^{2}=\lambda^{2} d z d \bar{z}$ of constant negative curvature which is induced by the noneuclidean metric on the unit disc, i.e. $2|d z| / 1-|z|^{2}$. We now write the operators introduced in Definition 1.2 for the holomorphic tangent bundle $\theta$ of $X$. In this case, we note that if $\phi=$ $b d z^{p} \wedge d \bar{z}^{q} \partial / \partial z \in C^{p, q}(X, \theta)$ then $b$ can be thought of as a $C^{\infty}$ function on $\Delta$ such that $b(\gamma(z))\left(\gamma^{\prime}\right)^{p-1}(z) \bar{\gamma}^{\prime}(z)^{q}=b(z)$ for all $\gamma \in \Gamma$. Here $0 \leq p \leq 1,0 \leq q \leq 1$. The volume element with respect to the metric $d s^{2}$ is $d X=(i / 2) \lambda^{2} d z \wedge d \bar{z}$. If $f$ is any differentiable function, then by $f_{z}, f_{\bar{z}}, f_{z \bar{z}}$, etc., we denote the derivatives $\partial f / \partial z, \partial f / \partial \bar{z}, \partial^{2} f / \partial z \partial \bar{z}$, etc.

$$
\begin{aligned}
& \bar{\partial} \phi=(-1)^{p} b_{\bar{z}} d z^{p} \wedge d \bar{z}^{1+q} \partial / \partial z, \\
& * \phi=(-1)^{p} i\left(\lambda^{2} / 2\right)^{1-p-q} b d z^{1-q} \wedge d z^{-1-p} \partial / \partial z, \\
& \# \phi=\left[(-1)^{p q} \lambda^{2} \bar{h} d z^{q} \wedge d \bar{z}\right] d z,
\end{aligned}
$$

(this notation indicates that \# $\phi$ is a $C^{\infty}$ form of type $(q, p)$ with values in $\theta^{*}$ )

$$
\delta \phi=(-1)^{p+q} 2 \lambda^{2(p+q-3)}\left(\lambda^{2(2-p-q)} b\right) z z^{p} \wedge d \bar{z}^{q-1} \partial / \partial z .
$$

Of course, the above formulas are to be understood with convention that a form containing $d z^{s}$ or $d \bar{z}^{r}$, with $s, r>1$, or $s<0$, is zero.

$$
\|\phi\|^{2}=\int_{X} i 2^{p+q-1} \lambda^{2(2-p-q)}|b|^{2} d z \wedge d \bar{z} .
$$

Proposition 1.6. The tangent bundle $\theta$ is $W^{0,0}$-elliptic with respect to the metric $d s^{2}=\lambda^{2} d z d \bar{z}$.

Proof. Let $\phi=b \partial / \partial z \in \mathfrak{D}^{0,0}(X, \theta)$. Then, using the formulas (2)-(5) we obtain the following expressions:

$$
\begin{aligned}
\square \phi & =\delta \bar{\partial} \phi=-2 \lambda^{-4}\left(\lambda^{2} b_{\bar{z}}\right) z / \partial z, \\
{ }^{*-1} \square * \phi & =-2 \lambda^{-2}\left[\lambda^{-2}\left(\lambda^{2} b\right)_{z}\right]_{\bar{z}} \partial / \partial z .
\end{aligned}
$$


Then

$$
\square \phi-{ }^{*-1} \square * \phi=2 \lambda^{-2}\left(2 \lambda^{-1} \lambda_{z \bar{z}}-2 \lambda^{-2} \lambda_{z} \lambda_{\bar{z}}\right) b \partial / \partial z .
$$

Since the metric $d s^{2}=\lambda^{2} d z d \bar{z}$ has curvature -1 ,

$$
\lambda^{2}=4(\log \lambda)_{z \bar{z}}=4\left(\lambda^{-1} \lambda_{z \bar{z}}-\lambda^{-2} \lambda_{z} \lambda_{\bar{z}}\right) .
$$

So $\square \phi-*^{-1} \square_{*} \phi=\phi$. Since $*^{-1}$ is the adjoint of $*$ and $\delta$ is the adjoint of $\bar{\partial}$ with respect to the $L_{2}$-scalar product, we get that

$$
\begin{aligned}
(\phi, \phi) & =(\square \phi, \phi)-\left(*^{-1} \square * \phi, \phi\right) \\
& =(\delta \bar{\partial} \phi, \phi)-(\bar{\partial} \delta * \phi, * \phi)=(\bar{\partial} \phi, \bar{\partial} \phi)-(\delta * \phi, \delta * \phi) .
\end{aligned}
$$

So $\|\phi\|^{2}=\|\bar{\partial} \phi\|^{2}-\|\delta * \phi\|^{2}$, or $\|\phi\|^{2} \leq\|\bar{\partial} \phi\|^{2}$. Q.E.D.

2. Complex structures. In this section we give the facts we need concerning almost complex and complex structures on a complex manifold. The proofs can be found in [9].

Let again $X$ be a complex manifold of dimension $n$ and let $X$ be the underlying $C^{\infty}$ manifold of dimension $2 n$. Let $\theta$ be the holomorphic tangent bundle of $X$ and $T \mathbf{X}$ the (real) tangent bundle of $X$. If $C T \mathbf{X}$ is the complexification of $T \mathbf{X}$, then $C T \mathbf{X}=\theta \oplus \bar{\theta}$, where $\bar{\theta}$ is the complex conjugate of $\theta$.

2.1. Definition. We say that we have an almost complex structure on $X$ if there is a $C^{\infty}$ vector subbundle $\theta^{\prime}$ of $C T \mathbf{X}$ (over $\mathbf{C}$ ) such that $C T \mathbf{X}=\theta^{\prime} \oplus \bar{\theta}^{\prime}$.

We note that a complex structure $X^{\prime}$ on $X$ determines an almost complex structure, namely the $C^{\infty}$ vector subbundle $\theta^{\prime}$, where in this case $\theta^{\prime}$ is the holomorphic tangent bundle of $X^{\prime}$. Let $\rho^{\prime \prime}(X)$ be the projection of CT $\mathbf{X}$ onto $\bar{\theta}$.

2.2. Definition. Let $\theta^{\prime}$ be an almost complex structure on $X$. We say that it is of finite distance from the given complex structure $X$ on $X$ when $\rho^{\prime \prime}(X)$ induces an isomorphism from $\bar{\theta}^{\prime}$ onto $\bar{\theta}$.

Proposition 2.3. There is a bijective correspondence between the set of almost complex structures of finite distance from $X$ and the set of all $C^{\infty}$ differential forms $\omega$ of type $(0,1)$ with values in $\theta$ such that, at each point $p \in X, \bar{\omega} \circ \bar{\omega}$ : $\bar{\theta}_{p} \rightarrow \bar{\theta}_{p}$ does not bave eigenvalue 1 .

We explain the last part of the proposition. Namely, every $C^{\infty}$ form $\omega$ of type $(0,1)$, with values in $\theta, \omega_{i}=\Sigma_{\alpha, \beta} \omega_{\alpha}^{\beta}\left(z_{i}\right) d \bar{z}_{i}^{\alpha} \partial / \partial z_{i}^{\beta}$ on the $i$ th coordinate neighborhood, can be thought of as a $C^{\infty}$ homomorphism $\omega: \bar{\theta} \rightarrow \theta$, given by $\omega\left(\Sigma_{a} \phi^{a} \partial / \partial \bar{z}^{a}\right)=$ $\Sigma_{a, \beta} \omega_{\alpha}^{\beta} \bar{\phi}{ }^{\alpha} \partial / \partial z^{\beta}$. One checks easily that this homomorphism is well defined because $\omega$ satisfies the right invariance property, $\omega_{i}=J_{i j} \omega_{j}$. At $p \in X$, we define $\bar{\omega}: \bar{\theta}_{p} \rightarrow \bar{\theta}_{p}$ by $\bar{\omega}(L)=\overline{\omega(L)}, L \in \bar{\theta}_{p}$.

Remark 2.4. Let $X$ be a Riemann surface. Then $\omega=w(z) d \bar{z} \partial / \partial z$ and, for 
$p \in X, \bar{\omega}: \bar{\theta}_{p} \rightarrow \bar{\theta}_{p}$ is given by $\bar{\omega}(\bar{b} \partial / \partial \bar{z})=\overline{w(z)} b(z) \partial / \partial \bar{z}$. Furthermore, $\bar{\omega} \circ \bar{\omega}(b \partial / \partial \bar{z})=\omega(\overline{w(z)} b(z) \partial / \partial \bar{z})=|\dot{w}|^{2} \bar{b} \partial / \partial \bar{z}$. Therefore, the condition $\sup _{X}|w(z)|$ $<1$ implies that $\omega$ determines an almost complex structure of finite distance from $X$.

The almost complex structure determined by $\omega$ will be denoted by $X_{\omega}$.

Theorem 2.5 (Newlander-Nirenberg). The almost complex structure $X_{\omega}$ is induced by a complex structure if and only if $\bar{\partial} \omega-1 / 2[\omega, \omega]=0$.

Here, $[\omega, \omega]=\Sigma_{\alpha, \beta, \gamma} \omega_{\beta \gamma}^{\alpha} d \bar{z}^{\beta} \wedge d \bar{z}^{\gamma} \partial / \partial z^{a}$, where

$$
\omega_{\beta \gamma}^{a}=\sum_{r}\left(\left(\partial \omega_{\gamma}^{a} / \partial z^{r}\right) \omega_{\beta}^{r}-\left(\partial \omega_{\beta}^{a} / \partial z^{r}\right) \omega_{\gamma}^{r}\right) .
$$

We note that $[\omega, \omega] \in C^{0,2}(X, \theta)$.

Remark 2.6. Let again $X$ be a Riemann surface. Then $\omega=w(z) d \bar{z} \partial / \partial z \epsilon$ $C^{0,1}(X, \theta)$ with $\sup _{X}|w(z)|<1$ determines a complex structure on $X$.

Let $f$ be a diffeomorphism of $X$. Let $\theta_{1}^{\prime}$ and $\theta_{2}^{\prime}$ be almost complex structures on $\mathrm{X}$.

2.7. Definition. We say that $f$ is an isomorphism between $\theta_{1}^{\prime}$ and $\theta_{2}^{\prime}$ if its differential $d f$ sends $\theta_{1}^{\prime}$ to $\theta_{2}^{\prime}$.

If $\omega \in C^{0,1}(X, \theta)$ such that $X_{\omega}$ can be defined, then there is a unique almost complex structure $\theta^{\prime}$ such that $f$ is an isomorphism of $\theta^{\prime}$ onto $X_{\omega} . \theta^{\prime}$ is induced by an element $\psi \in C^{0,1}(X, \theta)$ when $\theta^{\prime}$ is of finite distance from $X$. We set $\psi=\omega \circ f . \psi$ is called the transform of $\omega$ by $f . \omega \circ f$ can be defined if $f$ is sufficiently close to the identity map of $X$ in $C^{\prime}$-topology.

Let $z=\left(z^{1}, \ldots, z^{n}\right)$ be a chart of $X$ with domain $U$. Let $U_{1}$ be an open subset of $U$. Assuming that $f\left(U_{1}\right) \subseteq U$ and that $\omega \circ f=\psi$ can be defined in this case we have the following

Proposition 2.8. If $f(z)=\left(f^{1}(z), \ldots, f^{n}(z)\right), \omega=\Sigma \omega_{\alpha}^{\beta}(z) d \bar{z}^{a} \partial / \partial z^{\beta}, \psi=$ $\Sigma \psi_{\alpha}^{\beta}(z) d \bar{z}^{\alpha} \partial / \partial z^{\beta}$, then

$$
\sum_{\beta} \psi_{a}^{\beta}(z)\left(\frac{\partial f^{\gamma}}{\partial z^{\alpha}}+\sum_{\mu} \frac{\partial \bar{f}^{\mu}}{\partial z^{\beta}} \omega_{\mu}^{\gamma}(f(z))\right)=\frac{\partial f^{\gamma}}{\partial \bar{z}^{\alpha}}+\sum_{\mu} \frac{\partial \bar{f}^{\mu}}{\partial \bar{z}^{\alpha}} \omega_{\mu}^{\gamma}(f(z)) .
$$

We will also need the following fact:

Proposition 2.9. Let $V$ be a vector space and let $b: V \rightarrow C^{0,0}(X, \theta)$ be an R-linear map. Let $f^{s}$ be a $C^{\infty}$ family of diffeomorphisms of $\mathbf{X}$ such that $f^{0}$ is the identity map. Assume that, for each chart $z=\left(z^{\prime}, \ldots, z^{n}\right)$ on $X$,

$$
f_{a}^{s}(z) \equiv z^{a}+b^{a}(z, s) \quad\left(\bmod |s|^{2}\right) \quad(s \in V,|s| \text { small })
$$

where $b(s)=\Sigma_{a} b^{a}(z, s) \partial / \partial z^{a}$. Let $\omega$ be an element of $C^{0,1}(X, \theta)$ which represents a complex structure on $\mathrm{X}$, i.e. $\bar{\partial} \omega-1 / 2[\omega, \omega]=0$. Then 


$$
\omega \circ f^{s} \equiv \omega+\bar{\partial}_{\omega}(b(s)+\omega \circ \overline{b(s)})\left(\bmod |s|^{2}\right)
$$

Here $\bar{\partial}_{\omega} \eta=\bar{\partial} \eta-[\omega, \eta]$, where if $\eta=\Sigma \eta^{\alpha}(z) \partial / \partial z^{a} \in C^{0,0}(X, \theta)$ then

$$
[\omega, \eta]=\sum_{\alpha, \beta, \gamma}\left(\frac{\partial \eta^{\beta}}{\partial z^{\gamma}} \omega_{\alpha}^{\gamma}-\frac{\partial \omega_{a}^{\beta}}{\partial z^{\gamma}} \eta_{\gamma}\right)\left(\frac{\partial}{\partial z^{\beta}}\right) d \bar{z}^{\alpha} .
$$

If $\xi=\Sigma \xi^{\alpha}(z) \partial / \partial \bar{z}^{\alpha}$, then $\omega \circ \xi=\Sigma \omega_{\alpha}^{\beta} \xi^{\alpha} \partial / \partial z^{\beta}$.

3. Families of complex structures on Riemann surfaces. Proof of the main theorem. Throughout this section we assume that $X$ is a nonexceptional Riemann surface satisfying the following condition: the length of every closed geodesic is bounded from below.

Let $\Delta=\{z \in C|| z \mid<1\}$ and $\Omega \subset \Delta$ be a fundamental domain for $X$. Let $k>$ 0 be an integer.

3.1. Definition. If $\phi=b \lambda / \partial z \in C^{0,0}(X, \theta)$, let

$$
|\phi|_{k, \Omega}=\left(\sum_{a \leq k} \int_{\Omega}\left(1-|z|^{2}\right)^{2(a-2)}\left|D^{a} b\right|^{2} d x d y\right)^{1 / 2} .
$$

If $\omega=w d \bar{z} \partial / \partial z \in C^{0,1}(X, \theta)$, let

$$
|\omega|_{k, \Omega}=\left(\sum_{a \leq k} \int_{\Omega_{i}}\left(1-|z|^{2}\right)^{2(a-1)}\left|D^{a} w\right|^{2} d x d y\right)^{1 / 2} .
$$

Here $D^{\alpha}=\partial^{\alpha} \mid \partial z^{\alpha_{1}} \partial \bar{z}^{a_{2}}, \alpha=\alpha_{1}+\alpha_{2}$. It is easy to see that ||$_{k, \Omega}$ defines a norm.

Proposition 3.2. The equivalence class of this norm is independent of $\Omega$.

Proof. Let $\Omega^{\prime}$ be another fundamental domain. Then $\Omega^{\prime}=\gamma(\Omega)$, where $\gamma \epsilon$ $\Gamma, X=\Delta / \Gamma$. Let $\phi \in C^{0,0}(X, \theta)$ and assume $|\phi|_{k, \Omega}<\infty$. Then $b \circ \gamma=b \cdot \gamma^{\prime}$ for all $\gamma \in \Gamma$. If $\gamma=e^{i \theta}(z-\beta) /(1-\bar{\beta} z),|\beta|<1$, then we claim that

$$
D^{\alpha} b \circ \gamma=\left(\gamma^{\prime}\right)^{1-\alpha} \sum_{\nu=0}^{a} c_{\nu, \alpha}(1-\bar{\beta} z)^{\nu-\alpha} D^{\nu} b,
$$

where the $c_{\nu, a}$ depend only on $\alpha$. The claim is easily verified by induction on a. Now $1-|\gamma(z)|^{2}=\left(1-|z|^{2}\right)\left|\gamma^{\prime}(z)\right|$ and $|1-\bar{\beta} z| \geq 1-|z|$. On the other hand, we have

$$
|\phi|_{k, \Omega^{\prime}}^{2}=\sum_{\alpha<k} \int_{\Omega^{\prime}}\left(1-|\zeta|^{2}\right)^{2(\alpha-2)}\left|D^{\alpha} b(\zeta)\right|^{2} d \xi d \eta
$$


where $\zeta=\gamma(z), z \in \Omega$. So

$$
\begin{aligned}
\int_{\Omega^{\prime}}( & \left(\left.1 \zeta\right|^{2}\right)^{2(a-2)}\left|D^{\alpha} b(\zeta)\right|^{2} d \xi d \eta \\
& =\frac{i}{2} \int_{\Omega}\left(1-|\gamma(z)|^{2}\right)^{2(a-2)}\left|D^{\alpha} b \circ \gamma\right|^{2}\left|\gamma^{\prime}(z)\right|^{2} d z d \bar{z} \\
& \leq c \int_{\Omega}\left[\left(1-|\gamma(z)|^{2}\right) /\left|\gamma^{\prime}(z)\right|\right]^{2(a-2)} \sum_{\nu=0}^{a}\left|c_{\nu, a}\right|^{2}\left(1-|z|^{2}\right)^{2(\nu-d)}\left|D^{\nu} b\right|^{2} d x d y \\
& =c \int_{\Omega} \sum_{\nu=0}^{a}\left|c_{\nu, a}\right|^{2}\left(1-|z|^{2}\right)^{2(\nu-2)}\left|D^{\nu} b\right|^{2} d x d y .
\end{aligned}
$$

But this shows that $|\phi|_{k, \Omega^{\prime}}^{2} \leq c_{1}(k)|\phi|_{k, \Omega}^{2}$ In the same way we can prove $|\phi|_{k, \Omega}^{2}$ $\leq c_{2}(k)|\phi|_{k, \Omega^{\prime}}^{2}$. Here $c_{1}$ and $c_{2}$ depend only on $k$. Also, following the above method, we prove that there exist constants $c_{1}^{\prime}(k)$ and $c_{2}^{\prime}(k)$ such that $|\omega|_{k, \Omega^{\prime}} \leq$ $c_{1}^{\prime}(k)|\omega|_{k, \Omega},|\omega|_{k, \Omega} \leq c_{2}^{\prime}(k)|\omega|_{k, \Omega^{\prime}}$. Q.E.D.

Remark 3.2.1. From now on we will write $|\phi|_{k}$ and $|\omega|_{k}$ instead of $|\phi|_{k, \Omega}$ and $|\omega|_{k, \Omega}$. Let $C_{k}^{0}=\left\{\left.\phi \in C^{0,0}(X, \theta)|| \phi\right|_{k}<\infty\right\}$ and $C_{k}^{1}=\left\{\left.\omega \in C^{0,1}(X, \theta)|| \omega\right|_{k}\right.$ $<\infty\}$. By $\mathcal{H}_{k}^{0}$ and $\mathcal{H}_{k}^{1}$ we denote the Banach spaces, obtained by completing $C_{k}^{0}$ and $C_{k}^{1}$, resp., with respect to ||$_{k}$.

Remark 3.2.2. The proof of Proposition 3.2 shows that, if $A \subset \Omega$ is a measurable set and $A^{\prime}=\gamma(A) \subset \Omega^{\prime},|\phi|_{k, A^{\prime}} \leq c_{1}(k)|\phi|_{k, A},|\phi|_{k, A} \leq c_{2}(k)|\phi|_{k, A^{\prime}}$, where

$$
|\phi|_{k, A}^{2}=\sum_{a \leq k} \int_{A}\left(1-|z|^{2}\right)^{2(a-2)}\left|D^{a} b\right|^{2} d x d y
$$

Of course, the same thing holds for $\omega$, too.

We now prove the continuity of certain operators with respect to the ||$_{k}$-norm.

Proposition 3.3. There exists a constant $c_{3}(k)$ (depending only on $k$ ) such that if $\omega \in C_{k}^{1}$ then $|\delta \omega|_{k-1} \leq c_{3}(k)|\omega|_{k}$.

Proof. By (5) of $\$ 1$ with $p=0, q=1, \delta \omega=-2 \lambda^{-4}\left(\lambda^{2} w\right) z / \partial z, \omega=w d \bar{z} \partial / \partial z$. Put $b=-2 \lambda^{-4}(\partial / \partial z)\left(\lambda^{2} w\right)$. On $\Delta$,

$$
\begin{aligned}
\lambda & =2 /\left(1-|z|^{2}\right), \\
b & =-1 / 2\left(1-|z|^{2}\right)^{2} w_{z}-\bar{z}\left(1-|z|^{2}\right) w, \\
D^{a} b & =\sum_{\beta=0}^{a} d_{\alpha, \beta} D^{\beta}\left[\left(1-|z|^{2}\right)^{2}\right] D^{\alpha-\beta+1} w+\sum_{\beta=0}^{\alpha} d_{\alpha, \beta}^{\prime} D^{\beta}\left[\bar{z}\left(1-|z|^{2}\right)\right] D^{a-\beta} w,
\end{aligned}
$$

where $d_{\alpha, \beta}, d_{\alpha, \beta}^{\prime}$ are some constants. Now 


$$
\begin{aligned}
& \left(1-|z|^{2}\right)^{\alpha-2}\left|D^{\beta}\left[\left(1-|z|^{2}\right)^{2}\right]\right| \begin{cases}=\left(1-|z|^{2}\right)^{\alpha} & \text { if } \beta=0, \\
\leq 2\left(1-|z|^{2}\right)^{\alpha-1} & \text { if } \beta=1, \\
\leq \text { const. }\left(1-|z|^{2}\right)^{\alpha-2} & \text { if } \beta \geq 2 ;\end{cases} \\
& \left(1-|z|^{2}\right)^{\alpha-2}\left|D^{\beta}\left[\bar{z}\left(1-|z|^{2}\right)\right]\right|\left\{\begin{array}{l}
\leq \text { const. }\left(1-|z|^{2}\right)^{\alpha-\overline{1}} \text { if } \beta=0, \\
\leq \text { const. }\left(1-|z|^{2}\right)^{\alpha-2} \text { if } \beta \geq 1 .
\end{array}\right.
\end{aligned}
$$

Then

$$
\begin{aligned}
(1-|z|)^{a-2}\left|D^{a} b\right| \leq C_{2}^{\prime}(k) & {\left[\left(1-|z|^{2}\right)^{a}\left|D^{a+1} w\right|+\left(1-|z|^{2}\right)^{a-1}\left|D^{a} w\right|\right.} \\
& +\sum_{|\beta|=2}^{a}\left(1-|z|^{2}\right)^{\alpha-2}\left|D^{a-\beta+1} w\right| \\
& \left.+\sum_{\beta=1}^{a}\left(1-|z|^{2}\right)^{a-2}\left|D^{\alpha-\beta} w\right|\right] .
\end{aligned}
$$

On the other hand, $\alpha-2 \geq \alpha-\beta$ if $\beta \geq 2$ and $\alpha-2 \geq \alpha-\beta-1$ if $\beta \geq 1$. So (since $1-|z|^{2} \leq 1$ )

$$
\left(1-|z|^{2}\right)^{\alpha-2} \begin{cases}\leq\left(1-|z|^{2}\right)^{\alpha-\beta} & \text { if } \beta \geq 2, \text { and } \\ \leq\left(1-|z|^{2}\right)^{\alpha-\beta-1} & \text { if } \beta \geq 1 .\end{cases}
$$

Hence

$$
\left(1-|z|^{2}\right)^{\alpha-2}\left|D^{\alpha} b\right| \leq c_{3}^{\prime \prime}(k) \sum_{\beta=0}^{\alpha+1}\left(1-|z|^{2}\right)^{\beta-1}\left|D^{\beta} w\right|,
$$

which shows that $|\delta \omega|_{k-1} \leq c_{3}(k)|\omega|_{k}$. Q.E.D.

Proposition 3.4. If $\phi \in C_{k}^{0}(X, \theta)$, then there is a constant $c_{k}$, depending only on $k$, such that $|G \phi|_{k+2} \leq c_{k}|\phi|_{k}$.

Proof. By Theorem 1.4, $|G \phi|_{0}^{2}+|\bar{G} \phi|_{0}^{2} \leq$ const. $|\phi|_{0}$. Take a point $p_{0} \in X$ and let $\rho(p)=d\left(p_{0}, p\right)$ where $d\left(p_{0}, p\right)$ is the geodesic distance from $p_{0}$ to $p$. Then, in terms of local coordinates, $\rho$ is a locally Lipschitz function on $X$, and, whenever $\partial \rho / \partial z$ exists, we have $\left|\lambda^{-1}(z) \partial \rho / \partial z\right|=1 / 2, \lambda|d z|$ being the complete Riemannian metric on $X$ (cf. [3, p. 90]). This is easily verified on $\Omega$. For, if $z_{0}$ $\epsilon \Omega$ is such that $\pi\left(z_{0}\right)=p_{0}$, then 


$$
\rho(z)=\log \frac{1+\left|\left(z-z_{0}\right) /\left(1-\bar{z}_{0} z\right)\right|}{1-\left|\left(z-z_{0}\right) /\left(1-\bar{z}_{0} z\right)\right|} .
$$

Let $\sigma(z)=\left(z-z_{0}\right) /\left(1-\bar{z}_{0} z\right)$. Then $\left|\rho_{z}\right|=\left|\sigma^{\prime}(z)\right| /\left(1-|\sigma(z)|^{2}\right)=1 /\left(1-|z|^{2}\right)$ and $\left(1-|z|^{2}\right)\left|\rho_{z}\right|=1$.

Let $\nu(x)$ be a $C^{\infty}$ real valued function of the real variable $x$ such that

$$
\nu(x)=\left\{\begin{array}{ll}
1, & x \leq 1, \\
0, & x \geq 2,
\end{array} \text { and } 0 \leq \nu \leq 1 .\right.
$$

Then the function $\mu_{n}(p)=\nu(\rho / n)$ is a locally Lipschitz function $X$ such that $\mu_{n}=1$ on the geodesic ball $B_{n}$ with center $p_{0}$ and radius $n$ and $\mu_{n} \equiv 0$ outside the geodesic ball $B_{2 n}$.

Let $G \phi=\xi \partial / \partial z$. Now $\bar{\xi}\left(\lambda^{2} \xi\right)_{z} d z$ is a $C^{\infty}(1,0)$-form on $X$. Then $\mu_{n}^{2} \bar{\xi}\left(\lambda^{2} \xi\right)_{z} d z$ is a Lipschitz $(1,0)$-form on $X$ with compact support and, by Stokes' theorem, $\int_{X} d\left(\mu_{n}^{2} \bar{\xi}\left(\lambda^{2} \xi\right)_{z} d z\right)=0$, or, taking a fundamental domain $\Omega$, $\int_{\Omega} d\left[\mu_{n}^{2} \bar{\xi}\left(4 \xi\left(1-|z|^{2}\right)^{-2}\right)_{z} d z\right]=0$, where

$$
d\left(\mu_{n}^{2} \bar{\xi}\left(4 \xi\left(1-|z|^{2}\right)^{-2}\right)_{z} d z\right)=-\left[\mu_{n}^{2} \bar{\xi}\left(4 \xi\left(1-|z|^{2}\right)^{-2}\right)_{z}\right]_{\bar{z}} d z \wedge d \bar{z} .
$$

Differentiating the above expression and using the fact that, for any two functions $f$ and $g, \int|f g| \leq 1 / 2 \int|f|+1 / 2 \int|g|$, we obtain the following equality:

$$
\begin{aligned}
& \int_{\Omega} \mu_{n}^{2}\left|\xi_{z}\right|^{2}\left(1-|z|^{2}\right)^{-2} d x d y \\
& \quad \leq C_{0}^{\prime \prime}\left[\int_{\Omega}|\xi|^{2}\left(1-|z|^{2}\right)^{-4} d x d y+\int_{\Omega}\left|\xi_{\bar{z}}\right|^{2}\left(1-|z|^{2}\right)^{-2} d x d y+\int_{\Omega}\left|\xi_{z \bar{z}}\right|^{2} d x d y\right]
\end{aligned}
$$

for some constant $C_{0}^{\prime \prime}$. Since

$$
-1 / 2\left(1-|z|^{2}\right)^{2} \xi_{z \bar{z}}-\bar{z}\left(1-|z|^{2}\right) \xi_{\bar{z}}=b \quad(\phi=b \partial / \partial z)
$$

we see that there is a constant $c_{0}^{\prime}$ such that $|G \phi|_{1} \leq c_{0}^{\prime}|\phi|_{0}$.

We now proceed by induction: Assume that there is a constant $c_{k-1}$ such that $|G \phi|_{k+1} \leq c_{k-1}|\phi|_{k-1}$. Because of (*), we can estimate

$$
\int_{\Omega}\left(1-|z|^{2}\right)^{2 k}\left|\frac{\partial^{k+2} \xi}{\partial z^{\tau} \partial \bar{z}^{\sigma}}\right|^{2} d x d y
$$

in terms of

$$
\begin{array}{ll}
\int_{\Omega}\left(1-|z|^{2}\right)^{2(\alpha-2)}\left|D^{\alpha} b\right|^{2} d x d y & (\alpha \leq k), \\
\int_{\Omega}\left(1-|z|^{2}\right)^{2(\beta-2)}\left|D^{\beta} \xi\right|^{2} d x d y & (\beta \leq k+1) .
\end{array}
$$

Here $\tau+\sigma=k+2, \tau>0, \sigma>0$. Now, using 


$$
\begin{aligned}
& \int_{\Omega} d \mu_{n}^{2}\left(\lambda^{2}\right)^{k+1} \underbrace{\left(\lambda^{-2}\left(\lambda^{-2} \ldots\left(\lambda^{-2} \bar{\xi}_{z}\right)_{z} \cdots\right)_{z} z\right.}_{k} \\
& \left.\frac{\left(\lambda ^ { - 2 } \left(\lambda ^ { - 2 } \cdots \left(\lambda^{-2}\right.\right.\right.}{k+1} \frac{\left.\left.\left.\xi_{\bar{z}}\right)_{\bar{z}} \cdots\right)_{\bar{z}}\right)_{\bar{z}}}{k+2} d \bar{z}\right\}=0, \\
& \int_{\Omega} d\{\mu_{n}^{2} \lambda^{2 k} \underbrace{\left(\lambda^{-2}\left(\lambda^{-2} \cdots\left(\lambda^{-2} \bar{\xi}_{z}\right) \cdots\right)_{z}\right)}_{k} \\
& \left.\frac{\left(\lambda^{-2}\left(\lambda^{-2} \cdots\left(\lambda^{-2}\left(\lambda^{2} \xi\right)^{z}\right)_{\bar{z}} \cdots\right)_{\bar{z}}\right)_{\bar{z}}}{k+1} d z\right\}=0,
\end{aligned}
$$

we can estimate

$$
\int_{\Omega}\left(1-|z|^{2}\right)^{2 k}\left|\frac{\partial^{k+2} \xi}{\partial \bar{z}^{k+2}}\right|^{2} d x d y
$$

in terms of

$$
\int_{\Omega}\left(1-|z|^{2}\right)^{2 k}\left|\frac{\partial^{k+2} \xi}{\partial z^{\tau} \partial \bar{z}^{\sigma}}\right|^{2} d x d y, \quad \int_{\Omega}\left(1-|z|^{2}\right)^{2(\beta-2)}\left|D^{\beta} \xi\right|^{2} d x d y, \quad \beta \leq k+1 .
$$

Similarly, using

$$
\begin{gathered}
\int_{\Omega} d\left\{\mu_{n}^{2}\left(\lambda^{2}\right)^{k-1} \frac{\left(\lambda^{-2}\left(\lambda^{-2} \cdots\left(\lambda^{-2}\left(\lambda^{2} \bar{\xi}\right)_{\bar{z}}\right)_{\bar{z}} \cdots\right)_{\bar{z}}\right)_{\bar{z}}}{k+1}\right. \\
\left.\frac{\cdot\left(\lambda ^ { - 2 } \left(\lambda ^ { - 2 } \cdots \left(\lambda^{-2}\right.\right.\right.}{k+1} \frac{\left.\left.\left(\lambda^{2} \xi\right)_{z}\right)_{z} \cdots\right)_{\bar{z}}}{k+2}\right\} z=0, \\
\int_{\Omega} d\left\{\mu_{n}^{2}\left(\lambda^{2}\right)^{k-2} \frac{\left(\lambda^{-2}\left(\lambda^{-2} \cdots\left(\lambda^{-2}\left(\lambda^{2} \bar{\xi}\right)_{\bar{z}}\right)_{\bar{z}} \cdots\right)_{\bar{z}}\right)_{\bar{z}}}{k+1} \frac{\left(\lambda ^ { - 2 } \left(\lambda ^ { - 2 } \cdots \left(\lambda^{-2}\right.\right.\right.}{k} \frac{\left.\left.\left.\left(\lambda^{2} \xi\right)_{z}\right)_{\bar{z}} \cdots\right)_{z}\right)_{z}}{k+2}\right\}=0,
\end{gathered}
$$

we can obtain an estimate on $\int_{\Omega}\left(1-|z|^{2}\right)^{2 k}\left|\partial^{k+2} \xi / \partial z^{k+2}\right|^{2} d x d y$ so there is a constant $c_{k}$ such that $|G \phi|_{k+2} \leq c_{k}|\phi|_{k}$. Q.E.D.

Lemma 3.5. Let $t=\inf$ \{length $(\sigma): \sigma$ closed geod.\}. Let $0<s<t / 2$. For $z \in \Omega$, let $B(z, s)$ be a geodesic disc of radius $s$ and center $z$, i.e.

$$
B(z, s)=\left\{\zeta \in \Delta \mid \rho(z, \zeta)=\log \frac{1+|(z-\bar{\zeta}) /(1-\zeta z)|}{1-|(z-\zeta) /(1-\zeta z)|} \leq s\right\} \text {. }
$$


Let $\gamma_{1}, \cdots \gamma_{p} \in \Gamma$ be such that $B_{j}=B(z, s) \cap \stackrel{\circ}{\gamma}_{j}(\Omega) \neq \varnothing$ (bere ${ }^{\circ}$ stands for interior), $1 \leq j \leq p$. Then $\stackrel{\circ}{\gamma}_{j}^{-1}\left(B_{j}\right) \cap \stackrel{\circ}{\gamma}_{j}^{-1}\left(B_{i}\right)=\emptyset$ for $i \neq j$.

Proof. Assume not. Then there exists $\zeta \in \AA$ such that $\zeta=\gamma_{j}^{-1}\left(z_{j}\right)=\gamma_{i}^{-1}\left(z_{i}\right)$. First of all $z_{i} \neq z_{j}$ because $\stackrel{\circ}{\gamma}_{i}(\Omega) \cap \stackrel{\circ}{\gamma}_{j}(\Omega)=\emptyset$. Also $z_{j}=\gamma_{j} \gamma_{i}^{-1}\left(z_{i}\right), z_{j}, z_{i} \in B$. Thus $z_{i}$ and $z_{j}$ determine the same point on $X$. Moreover, the noneuclidean straight line connecting $z_{i}$ and $z_{j}$ corresponds to a closed geodesic on $X$ whose length is strictly less than $t$, because $z_{i}, z_{j} \in B$. But this is a contradiction. Q.E.D.

Proposition 3.5.1. There exists a constant $c_{4}(k)$ such that, for all $z \in \Omega$ and all $\phi=b \partial / \partial z \in C_{k}^{0},|b(z)| \leq c_{4}(k)\left(1-|z|^{2}\right)|\phi|_{k}$, for $k \geq 2$.

Proof. First we make the following observation: Let $u$ be a $C^{k}$-function with compact support on $\Delta$. Let $z \in \Delta$. Consider the function $\psi(r)=$ $(1 / 2 \pi) \int_{0}^{2 \pi} u\left(z+r e^{i \theta}\right) d \theta$. Then $\psi(0)=u(z)$ and $\psi$, as a function of one real variable, is also a $C^{k}$-function with compact support. By integration by parts we obtain that $u(z)=-\int_{0}^{\infty} r \psi^{\prime \prime}(r) d r$. Now

$$
\psi^{\prime \prime}(r)=\left.\frac{1}{2 \pi} \int_{0}^{2 \pi}\left(\frac{\partial^{2} u}{\partial \zeta^{2}} e^{2 i \theta}+2 \frac{\partial^{2} u}{\partial \zeta \partial \bar{\zeta}}+\frac{\partial^{2} u}{\partial \bar{\zeta}^{2}} e^{-2 i \theta}\right) d \theta\right|_{\zeta=z+r e^{i \theta}}
$$

So

$$
u(z)=\frac{1}{2 \pi} \int_{0}^{\infty} \int_{0}^{2 \pi} \frac{r}{|\zeta-z|^{2}}
$$

$$
\cdot\left[\frac{\partial^{2} u}{\partial \zeta^{2}}(\zeta-z)^{2}+2 \frac{\partial^{2} u}{\partial \zeta \partial \bar{\zeta}}(\zeta-z)(\bar{\zeta}-\bar{z})+\frac{\partial^{2} u}{\partial \bar{\zeta}^{2}}(\bar{\zeta}-\bar{z})^{2}\right] d r d \theta
$$

or

$$
u(z)=-\frac{i}{4 \pi} \int_{\Delta} \frac{1}{|\zeta-z|^{2}}
$$

$$
\cdot\left[\frac{\partial^{2} u}{\partial \zeta^{2}}(\zeta-z)^{2}+2 \frac{\partial^{2} u}{\partial \zeta \partial \bar{\zeta}}(\zeta-z)(\bar{\zeta}-\bar{z})+\frac{\partial^{2} u}{\partial \bar{\zeta}^{2}}(\bar{\zeta}-\bar{z})^{2}\right] d \zeta d \bar{\zeta}
$$

Take $r \leq t / 4$, where $t$ is as in Lemma 3.5. Let

$$
\rho(\zeta)=\log \frac{1+|(z-\zeta) /(1-\bar{\zeta} z)|}{1-|(z-\zeta) /(1-\bar{\zeta} z)|}
$$

and let $\nu$ be the function appearing in Proposition 3.4. Then $\mu(\zeta)=\nu(\rho(\zeta) / r)$ is identically 1 on $B(z, 2 r)$ and identically equal to 0 outside $B(z, 2 r)$. The function $\mu b$ is $C^{\infty}$ and with compact support in $\Delta$ and $\mu(z) b(z)=b(z)$. Now, $D^{2}(\mu b)$ $=\mu D^{2} b+2 D^{1} \mu D^{1} b+b D^{2} \mu$. By a direct calculation one verifies that $\left|D^{1} \mu\right| \leq$ 
$c_{4}^{\prime}\left(1-|\zeta|^{2}\right)^{-1} ;\left|D^{2} \mu\right| \leq c_{4}^{\prime}\left(1-|\zeta|^{2}\right)^{-2}$, where the constant $c_{4}^{\prime}$ depends on $r$, too. Formula (8), applied to the function $\mu h$, gives that

$$
\begin{aligned}
&|b(z)| \leq \text { const. } \int_{B(z, 2 r)}\left(\left|D^{2} b\right|+\left|D^{1} b\right|\left(1-|\zeta|^{2}\right)^{-1}+|b|\left(1-|\zeta|^{2}\right)^{-2}\right. \\
& \leq \text { const. }\left(\int_{B(z, 2 r)} d \zeta d \bar{\zeta}\right)^{1 / 2} \\
& \cdot\left(\int_{B(z, 2 r)}\left(\left|D^{2} b\right|^{2}+\left|D^{1} b\right|^{2}\left(1-|\zeta|^{2}\right)^{-2}+|b|^{2}\left(1-|\zeta|^{2}\right)^{-4}\right) d \zeta d \bar{\zeta}\right. \\
& \int_{B(z, 2 r)} d \zeta d \bar{\zeta}=\int_{\Delta_{r}}\left(1-|z|^{2}\right)^{2}|1+\bar{z} w|^{-4} d w d \bar{w}, \\
& w=\frac{\zeta-z}{1-\bar{z} \zeta} \text { and } \Delta_{r}=\left\{z \in \Delta /|z| \leq\left(e^{4 r}-1\right) /\left(e^{4 r}+1\right)\right\} .
\end{aligned}
$$

Let $\Omega_{1}, \Omega_{2}, \ldots, \Omega_{p}$ be fundamental domains such that $B_{j}=\Omega_{j} \cap B(z, 2 r) \neq$ $\emptyset, \Omega_{j}=\gamma_{j}(\Omega), \gamma_{j} \in \Gamma$. Then

$$
\begin{aligned}
\int_{B(z, 2 r)} & \left(\left|D^{2} b\right|^{2}+\left|D^{1} b\right|^{2}\left(1-|\zeta|^{2}\right)^{-2}+|b|^{2}\left(1-|\zeta|^{2}\right)^{-4}\right) d \zeta d \bar{\zeta} \\
& =\sum_{j=1}^{p} \int_{B_{j}}\left(\left|D^{2} b\right|^{2}+\left|D^{1} b\right|^{2}\left(1-|\zeta|^{2}\right)^{-2}+|b|^{2}\left(1-|\zeta|^{2}\right)^{-4}\right) d \zeta d \bar{\zeta}
\end{aligned}
$$

As in the proof of Proposition 3.2 we see that

$$
\begin{aligned}
& \frac{i}{2} \int_{B_{j}}\left(\left|D^{2} b\right|^{2}+\left|D^{1} b\right|^{2}\left(1-|\zeta|^{2}\right)^{-2}+|b|^{2}\left(1-|\zeta|^{2}\right)^{-4}\right) d \zeta d \bar{\zeta} \\
& \quad \leq \frac{i}{2} c_{4}^{\prime} \int_{\gamma_{j}^{-1}\left(B_{j}\right)}\left(\left|D^{2} b\right|^{2}+\left|D^{1} b\right|^{2}\left(1-\left|\zeta^{\prime}\right|^{2}\right)^{-2}+|b|^{2}\left(1-\left|\zeta^{\prime}\right|^{2}\right)^{-4}\right) d \zeta^{\prime} d \bar{\zeta}^{\prime} .
\end{aligned}
$$

By Lemma 3.5.,

$$
\begin{aligned}
& \sum_{j=1}^{p} \frac{i}{2} \int\left(\left|D^{2} b\right|^{2}+\left|D^{1} b\right|^{2}\left(1-\left|\zeta^{\prime}\right|^{2}\right)^{-2}+|b|^{2}\left(1-\left|\zeta^{\prime}\right|^{2}\right)^{-4}\right) d \zeta^{\prime} d \bar{\zeta}^{\prime} \\
& \leq \frac{i}{2} \int_{\Omega}\left(\left|D^{2} b\right|^{2}+\left|D^{1} b\right|^{2}\left(1-\left|\zeta^{\prime}\right|^{2}\right)^{-2}+|b|^{2}\left(1-\left|\zeta^{\prime}\right|^{2}\right)^{-4}\right) d \zeta^{\prime} d \bar{\zeta}^{\prime}
\end{aligned}
$$

(9), (10), and (11) imply that $|b(z)| \leq c_{4}(2)\left(1-|z|^{2}\right)|\phi|_{2}$. Q.E.D.

Corollary 3.5.2. There exists a constant $c_{5}(k)$ such that if $\phi=b \partial / \partial z \in C_{k}^{0}$ and $\omega=w d \bar{z} \partial / \partial z \in C_{k}^{1}$ then $\left|D^{a} b(z)\right| \leq c_{5}(k)\left(1-|z|^{2}\right)^{1-a}|\phi|_{k}$ and $\left|D^{\alpha} w(z)\right| \leq$ $c_{s}(k)\left(1-|z|^{2}\right)-a|\omega|_{k}$, where $k \geq a+2$.

Proof. Repeat the proof of Proposition 3.5.1 for the functions $\left(1-|z|^{2}\right)^{\alpha} D^{\alpha} b$ and $\left(1-|z|^{2}\right)^{a+1} D^{a} w$. Q.E.D.

Remark 3.5.3. The above inequalities are independent of $\Omega$. Using the relation 


$$
D^{\alpha} b \circ \gamma=\frac{1}{\left(\gamma^{\prime}\right)^{|a|-1}} \sum_{\nu=0}^{a} D^{\nu} b, \quad \gamma=e^{i \theta} \frac{z-\beta}{1-\bar{\beta} z} \in \Gamma,
$$

appearing in Proposition 3.2, we see that

$$
\left(1-|\gamma(z)|^{2}\right)^{a-1}\left|D^{\alpha} b \circ \gamma\right| \leq \sum_{\nu=0}^{a}\left|c_{\nu, a}\right|\left(1-|z|^{2}\right)^{\nu-1}\left|D^{\nu} b\right| \leq c_{5}^{\prime}(k)|\phi|_{k} .
$$

Of course, the same thing holds for $\omega=w d \bar{z} \partial / \partial z \in C_{k}^{1}$.

Let $\phi \in C_{k}^{0}$ and $p \in X$. We draw a geodesic $g(t)$ starting from the point $p$ in the direction of $\phi(p)$. Let $f(p)=g(1)$. Thus we obtain a differentiable mapping $f: X \rightarrow X$.

Lemma 3.6. If $|\phi|_{k}$ is sufficiently small, $f$ is a diffeomorphism.

Proof. In local coordinates the geodesic equation is

$$
\frac{d^{2} g(z, t)}{d t^{2}}=-2 \frac{\lambda_{z}(g(z, t))}{\lambda(g(z, t))}\left(\frac{d g(z, t)}{d t}\right)^{2}
$$

with the initial conditions

$$
g(z, 0)=z,\left.\quad \frac{d g(z, t)}{d t}\right|_{t=0}=b(z), \quad \phi=b \frac{\partial}{\partial z} .
$$

Then it is easy to see that $f$ is of the form $f(z)=z+\eta(z)$, where

$$
\eta(z)=b(z)+\sum_{n \geq 2} P_{n}(z) ; \quad P_{n}(z)=\lambda^{n-1}(z) \sum_{\mu+\nu=n} \epsilon_{\mu, \nu}(z) b^{\mu}(z) \bar{b}^{\nu}(z) .
$$

Here $\epsilon_{\mu, \nu}$ are bounded functions of $z$ and $\bar{z}$. By Proposition 3.5.1 and Corollary 3.5.2 it is clear that we can find a constant $c_{6}(k)$ such that $|\lambda(z) \eta(z)| \leq c_{6}(k)|\phi|_{k}$; $\left|D^{1} \eta(z)\right| \leq c_{6}(k)|\phi|_{k}$, if $|\phi|_{k}$ is sufficiently small. The Jacobian of $f$ is $\left|f_{z}\right|^{2}-$ $\left|f_{\bar{z}}\right|^{2}=\left|1+\eta_{z}\right|-\left|\eta_{\bar{z}}\right| \geq 1-2 c_{G}(k)|\phi|_{k}$, which is strictly positive if $|\phi|_{k}$ is sufficiently small. So $f$ is locally a diffeomorphism, which means that the image of $f$ is open. Let $p_{1}, \cdots, p_{n}, \cdots$ be points in the image and suppose that $\lim _{n \rightarrow \infty} p_{n}=p$. Let $q_{1}, \cdots, q_{n}, \cdots$ be such that $f\left(q_{j}\right)=p_{j}$. Now the sequence $\left\{q_{n}\right\}$ cannot be discrete because the length of the geodesic joining $p_{n}$ and $q_{n}$ is $\left|\lambda\left(q_{n}\right) b\left(q_{n}\right)\right|$ and $|\lambda(z) b(z)| \leq c_{6}|\phi|_{k}$. So there is $q$ such that $\lim q_{\nu_{n}}=q$. Thus $f(q)=\lim _{n \rightarrow \infty} f\left(q_{\nu_{n}}\right)=\lim _{\nu \rightarrow \infty} p_{\nu_{n}}=p$. Hence the image is also closed and $f$ is onto. We prove now that $f$ is one-to-one. Let $p_{1}, p_{2} \in X, p_{1} \neq p_{2}$. Let $t$ be as in Lemma 3.5 and assume $\phi$ is such that $|\phi|_{k} \leq t / 8 c_{6}(k)$. Then $f\left(p_{1}\right) \neq f\left(p_{2}\right)$ if $d\left(p_{1}, p_{2}\right)>t / 4$, where $d\left(p_{1}, p_{2}\right)$ is the geodesic distance between $p_{1}$ and $p_{2}$. This is so because $d\left(p_{1}, f\left(p_{1}\right)\right)=\left|\lambda\left(p_{1}\right) b\left(p_{1}\right)\right|, d\left(p_{2}, f\left(p_{2}\right)\right)=\left|\lambda\left(p_{2}\right) b\left(p_{2}\right)\right|$, and if $f\left(p_{1}\right)=f\left(p_{2}\right)$ then

$$
d\left(p_{1}, p_{2}\right) \leq d\left(p_{1}, f\left(p_{1}\right)\right)+d\left(f\left(p_{2}\right), p_{2}\right) \leq 2 c_{6}|\phi|_{k}=t / 4 .
$$


But this is a contradiction. So we consider the case when $d\left(p_{1}, p_{2}\right) \leq t / 4$. Let $B$ $=\left\{p \in X=d\left(p_{1}, p\right) \leq t / 2\right\}$ and let $\pi: \Delta \rightarrow X$. Then $\pi: \pi^{-1}(B) \rightarrow B$ is one-to-one. We consider the map $\pi^{-1} \circ f \circ \pi$ which we denote again by $f$. Now assume that $f\left(z_{1}\right)=f\left(z_{2}\right)$ where $z_{j}$ corresponds to $p_{j}$. Then $z_{1}+\eta\left(z_{1}\right)=z_{2}+\eta\left(z_{2}\right)$ and $\left|z_{1}-z_{2}\right|=\left|\eta\left(z_{1}\right)-\eta\left(z_{2}\right)\right| \leq\left(\left|\eta_{z}\left(z^{*}\right)\right|+\left|\eta_{\bar{z}}\left(z^{*}\right)\right|\right)\left|z_{1}-z_{2}\right| \leq 2 c_{6}(k)|\phi|_{k}\left|z_{1}-z_{2}\right|$, but this is impossible if $2 c_{6}(k)|\phi|_{k}<1$. So $f\left(p_{1}\right) \neq f\left(p_{2}\right)$. Q.E.D.

Remark 3.6.1. The diffeomorphism $f: X \rightarrow X$ can be lifted to a diffeomorphism of $\Delta$ onto itself which we denote by $f$, too. Since $|\eta(z)| \leq c_{6}(k)\left(1-|z|^{2}\right)|\phi|_{k}$, $\left.f\right|_{\partial \Delta}=$ id.

Let $\omega \in C_{k}^{\prime}$. If $|\omega|_{k}$ is sufficiently small then $\omega$ of can be defined, and by Proposition 2.8

$$
\omega \circ f(z)=\frac{f_{\bar{z}}+w(f(z)) \bar{f}_{\bar{z}}}{f_{z}+w(f(z)) \bar{f}_{z}} .
$$

Denote $\omega \circ f$ by $R(\omega, \phi)$.

Proposition 3.7. If $U$ and $W$ are sufficiently small neighborboods of 0 in $C_{k}^{1}$ and $C_{k}^{0}$, resp. ( $k$ sufficiently large), then $R: U \times W \rightarrow C_{k-1}^{1}$ is a uniformly continuous mapping.

Proof. First we make some general remarks. If $u$ is a $C^{k}$-function and if $D^{s}$ indicates differentiation of order $s<k$, we denote by $\left(D^{s} u\right)^{n}$ any expression of the form $\Pi_{j=1}^{n} \partial^{s} u / \partial z^{s}{ }_{1 j} \partial \bar{z}^{s}{ }^{s}, s_{1 j}+s_{2 j}=s$. If $v$ is also a $C^{k}$-function, then

$$
D^{s}(u \cdot v)=\sum_{k=0}^{s} c_{k} D^{s-k} u \cdot D^{k} v, \quad c_{k} \text { being constants. }
$$

$$
D^{s}(u / v)=\sum\left(c_{r_{1}} \ldots r_{n} / v^{n+1}\right) D^{r_{1}} v \ldots D^{r} v \cdot D^{s-n} u \text {, }
$$

where $c_{r_{1} \ldots r_{n}}$ are some constants and the summation is over $n, r_{1}, \cdots, r_{n}$ such that $0 \leq n \leq s$ and $r_{1}+\cdots+r_{n}=n$.

$$
D^{s}(u(v(z)))=\sum a_{r_{1}} \ldots r_{s}\left(D^{r} u\right)(v(z)) \cdot\left(D^{1} v\right)^{r_{1}} \ldots\left(D^{s} v\right)^{r^{s}},
$$

where $a_{r_{1} \ldots r_{s}}$ are constants and the summation is over all $r, r_{1}, \ldots, r_{s}$ such that $r_{1}+\cdots+r_{s}=r, r_{1}+2 r_{2}+\cdots+s r_{s}=s, 1 \leq r \leq s$.

These relations can trivially be checked by induction.

Furthermore, let $\phi \in C_{k}^{0}$ with $|\phi|_{k}$ sufficiently small and let $f=z+\eta(z)$ be as in Lemma 3.6. Then it can be easily verified that

$$
b_{1}\left(1-|z+t \eta|^{2}\right) \leq 1-|z|^{2} \leq b_{2}\left(1-|z+t \eta|^{2}\right)
$$

for some constants $b_{1}$ and $b_{2}$ and $0 \leq t \leq 1$. 
Finally, let $\omega_{j} \in C_{k}^{1}, \phi_{j} \in C_{k}^{0}, j=1,2$. Now, using Proposition 3.5 .1 and Corollary 3.5.2 together with (12), (13), (14) and (15), one can show that if $\omega_{j}$ and $\phi_{j}$ are sufficiently small in ||$_{k}$-norm, then

$$
\left|R\left(\omega_{1}, \phi_{1}\right)-R\left(\omega_{2}, \phi_{2}\right)\right|_{k-1} \leq \text { const. }\left(\left|\omega_{1}-\omega_{2}\right|_{k}+\left|\phi_{1}-\phi_{2}\right|_{k}\right) .
$$

We omit the details which can be carried out in a completely straightforward way. Q.E.D.

Remark 3.7.1. Propositions 3.3, 3.4 and 3.7 show that the mappings $\delta: C_{k}^{1}$ $\rightarrow C_{k-1}^{0}, G: C_{k}^{0} \rightarrow C_{k+2}^{0}$ and $R: U \times W \rightarrow C_{k-1}^{1}$ are uniformly continuous. Therefore we can consider them as continuous mappings between $\mathcal{H}_{k}^{1}$ and $\mathcal{H}_{k-1}^{0}, \mathcal{H}_{k}^{0}$ and $\mathcal{H}_{k+2}^{0}, U_{0} \times W_{0}$ and $\mathcal{H}_{k-1}^{1}$, resp., where $U_{0}$ and $W_{0}$ are neighborhoods of 0 in $\mathcal{H}_{k}^{1}$ and $\mathcal{H}_{k}^{0}$, resp.

Proposition 3.8. If $U_{0}$ and $W_{0}$ are sufficiently small then $R: U_{0} \times W_{0} \rightarrow$ $\mathcal{H}_{k-1}^{1}$ is a $C^{\infty}$ mapping.

Proof. We formally differentiate $R(\omega, \phi)$ with respect to the "variables" $w(f(z)), h, \bar{b}, b_{z}, \bar{h}_{z}, b_{\bar{z}}, \bar{h}_{\bar{z}}$ and denote the respective expressions by $D_{1} R, \cdots, D_{7} R$. If $\omega_{0} \in \mathcal{H}_{k}^{1}$ and $\phi_{0} \in \mathcal{H}_{k}^{0}$ with $\left|\omega_{0}\right|_{k}$ and $\left|\phi_{0}\right|_{k}$ sufficiently small, it can easily be shown (by using Proposition 3.5.1, Corollary 3.5.2, and the relations in Proposition 3.7) that the linear map $D R\left({ }_{0},{ }_{0}\right): \mathcal{H}_{k}^{1} \times \mathcal{H}_{k}^{0} \rightarrow \mathcal{H}_{k-1}^{1}$ defined by

$$
D R\left(\omega_{0}, \phi_{0}\right)(\omega, \phi)=D_{1} R\left(\omega_{0}, \phi_{0}\right) \cdot w+\cdots+D_{7} R\left(\omega_{0}, \phi_{0}\right) \cdot \bar{h}_{\bar{z}}
$$

$(w=w d \bar{z} \partial / \partial z, \phi=b \partial / \partial z)$ is continuous. By the same methods one can check that

$\left|R(\omega, \phi)-R\left(\omega_{0}, \phi_{0}\right)-D R\left(\omega_{0}, \phi_{0}\right)\left(\omega-\omega_{0}, \phi-\phi_{0}\right)\right|_{k-1}=o\left(\left|\omega-\omega_{0}\right|_{k}+\left|\phi-\phi_{0}\right|_{k}\right)$ provided that $\omega$ and $\phi$ are sufficiently small in the ||$_{k}$-norm. This shows that $R(\omega, \phi)$ is a differentiable mapping in the sense of the theory of Banach spaces with derivative $D R\left(\omega_{0}, \phi_{0}\right)$ at the point $\left(\omega_{0}, \phi_{0}\right)$. In the same way one can establish the existence of higher order derivatives. Again, as in Proposition 3.7, we have omitted most of the details because they can be filled in without any difficulties. Q.E.D.

Let now $H_{k}^{1}=\left\{\omega \in C_{k}^{1} \mid \delta \omega=0\right\}$. $H_{k}^{1}$ is a Banach subspace of $\mathcal{H}_{k}^{1}$. Let $B_{k}=$ $\left\{\omega=w(z) d \bar{z} \partial / \partial z \in H_{k}^{1}|\sup | w(z) \mid<1\right\}$. By Remark 2.6 all $\omega$ 's in $B_{k}$ determine complex structures on $X$. It is also obvious that $B_{k}$ is an open subset of $H_{k}^{1}$.

3.9. Definition. By a complex analytic family of deformations of $X$ we will mean a complex analytic map $\omega: S \rightarrow C_{k}^{1}$, where $S$ is an open subset of a Banach space, $\omega\left(s^{0}\right)=0$ for some $s^{0} \in S$, and $\sup |w(z, s)|<1$, where $\omega(s)=$ $w(z, s) d \bar{z} \partial / \partial z$. 
Remark 3.9.1. If $S$ is a subset of $\mathbf{C}^{n}$, then, for every fixed $z, w(z, s)$ is a complex analytic function of $s$.

Theorem 3.10 (Main Theorem). $B_{k}$ represents a universal family of deformations of $X$, i.e. if $\omega: S \rightarrow C_{k}^{1}$ is a complex analytic family of deformations, then there exists a complex analytic map $\tau: S^{\prime} \rightarrow B_{k}$, where $S^{\prime}$ is some neighborbood of $s^{0}$ in $S$, and a continuous family of diffeomorphisms $f^{s}$, depending differentiably on $s$, such that $r\left(s^{0}\right)=0, f^{s^{0}}=\mathrm{id}$ and $\omega(s) \circ f^{s}=\tau(s)$.

Proof. Let $P(\omega, \phi)=R(\omega, \phi)-\omega-\bar{\partial} \phi$. Then $P(\omega, \phi)$ is a function of $\omega$ and $\phi$ of order at least 2 in a sense that it contains terms of the form $w(z+\eta)^{\mu_{0}} b^{\mu_{1}} \bar{b}^{\mu_{2}} b_{\bar{z}} b_{\bar{z}}{ }^{\mu_{3} \bar{b}_{\bar{z}}}{ }^{\mu_{4}} b_{z}{ }^{\mu_{5} \bar{b}_{z}}{ }^{\mu_{6}}$ where $\mu_{0}+\mu_{1}+\cdots+\mu_{6} \geq 2 ; \omega(z)=$ $w(z) d \bar{z} \partial / \partial z, \phi(z)=b(z) \partial / \partial z$.

Now consider the mapping $T: S_{0}^{\prime} \times W_{0} \rightarrow \mathcal{H}_{k}^{0}$, defined by $T(s, \phi)=\phi+$ $G \delta \omega(s)+G \delta P(\omega(s), \phi)$. If $S_{0}^{\prime}$ and $W_{0}$ are sufficiently small neighborhoods of $s^{0}$ in $S$ and 0 in $\mathcal{H}_{k}^{0}$, resp., then Propositions 3.3, 3.4, 3.7 and 3.8 show that $T$ is a $C^{\infty}$ mapping. Moreover, the derivative of $T$ with respect to $\phi$ at $(0,0)$ is the identity and $T(0,0)=0$. Therefore, by the implicit function theorem for Banach spaces there exists a neighborhood $S^{\prime}$ of $s^{0}$ in $S$ and a unique $C^{\infty}$ mapping $s$ $\rightarrow \phi(s)$ of $s^{\prime}$ into $\mathcal{H}_{k}^{0}$ such that $\phi\left(s^{0}\right)=0$ and

$$
T(s, \phi(s))=\phi(s)+G \delta \omega(s)+G \delta P(\omega(s), \phi(s))=0 .
$$

On the other hand,

$$
\omega(s)+G \delta \omega(s)+G \delta P(\omega(s), \phi(s))=G(\delta \bar{\partial} \phi(s)+\delta \omega(s)+\delta P(\omega(s), \phi(s))) .
$$

Then, by Theorem 1.4 and Proposition 1.6, $T(s, \phi)=0$ if and only if $\delta \bar{\partial} \phi(s)+$ $\delta \omega(s)+\delta P(\omega(s), \phi(s))=0$. By Corollary 3.5.2 it follows that $\phi(s)$ is at least $C^{\alpha}$ for $a \leq k-2$. So if $k$ is sufficien tly large $(k \geq 3)$ then it makes sense to consider the map $f^{s}$ (see Lemma 3.6) corresponding to $\phi(s)$. On the other hand,

$$
\delta \bar{\partial} \phi(s)+\delta \omega(s)+\delta P(\omega(s), \phi(s))=\delta \omega(s) \circ f^{s} \text {. }
$$

Now $\delta \omega(s) \circ f^{s}=0$ implies $\bar{\partial} \omega \omega(s) \circ f^{s}=0$, and since it is a strongly elliptic differential equation it follows that $\omega(s) \circ f^{s}$ is $C^{\infty}$. So if $S^{\prime}$ is sufficiently small, $\omega(s) \circ f^{s}$ represents a complex structure on $X$. Since $f^{s}$ is a complex analytic isomorphism of $X_{\omega(s) \circ f^{s}}$ onto $X_{\omega(s)}($ see $\$ 2)$, it follows that $f^{s}$ is a diffeomorphism. Put $r(s)$ $=\omega(s) \circ f^{s}$. Then $\tau\left(s^{0}\right)=0$ and $f^{s^{0}}=\mathrm{id}$. It remains to prove that $\tau(s)$ is complex analytic if $S^{\prime}$ is sufficiently small. We note first that since $\phi(s)$ is continuously differentiable in $s$ so is $\tau(s)$. Furthermore, without loss of generality we may assume that $S \subset \mathbf{C}^{n}$. Let $s \in S^{\prime}$,

$$
\begin{aligned}
\tau(s+u)= & \omega(s+u) \circ f^{s+u}=\omega(s+u) \circ f^{s+u}-\omega(s) \circ f^{s+u}+\omega(s) \circ f^{s+u} \\
= & \left(\omega(s+u) \circ f^{s}-\omega(s) \circ f^{s}\right) \\
& +\underbrace{\omega(s+u) \circ f^{s+u}-\omega(s+u) \circ f^{s}+\omega(s) \circ f^{s}-\omega(s) \circ f^{s+u}}_{A}+\omega(s) \circ f^{s+u} .
\end{aligned}
$$


Since $\omega \circ f=\omega+\bar{\partial} \phi+P(\omega, \phi)$, we find that $A=O\left(|u|^{2}\right)$. Thus, $\tau(s+u)=$ $\left(\omega(s+u) \circ f^{s}-\omega(s) \circ f^{s}\right)+\omega(s) \circ f^{s+u}+O\left(|u|^{2}\right)$. Since

$$
\omega(s+u) \circ f^{s}=\frac{f_{\bar{z}}^{s}+w\left(f^{s}(z), s+u\right) \bar{f}_{\bar{z}}^{s}}{f_{z}^{s}+w\left(f^{s}(z), s+u\right) \bar{f}_{z}^{s}}
$$

we see that, for fixed $s, \omega(s+u) \circ f^{s}$ depends complex analytically on $u$ if $\omega(s+u)$ is complex analytic. Also, by Proposition 2.9 we have

$$
\omega(s) \circ f^{s+u}=\omega(s)+\bar{\partial}_{\omega(s)}(\phi(s+u)+\omega(s) \circ \overline{\phi(s+u)})+O\left(|u|^{2}\right) .
$$

Put $\chi(s+u)=\phi(s+u)+\omega(s) \circ \overline{\phi(s+u)}$. Here $\omega(s) \circ \overline{\phi(s+u)}=$ $w(z, s) \overline{b(z, s+u)} \partial / \partial z ; \omega(s)=w(z, s) d \bar{z} \partial / \partial z ; \phi(s+u)=b(z, s+u) \partial / \partial z$. Then

$$
\omega(s) \circ f^{s+u}=\omega(s)+\bar{\partial} \chi(s+u)-\left[\omega(s), \chi^{(s+u)]+O\left(|u|^{2}\right),}\right.
$$

where

$$
\begin{aligned}
{[\omega(s), \chi(s+u)] } & =\left[\frac{\partial \xi(z, s+u)}{\partial z} w(z, s)-\frac{\partial w(z, s)}{\partial z} \xi(z, s+u)\right] d \bar{z} \frac{\partial}{\partial z} \\
\chi(s+u) & =\xi(z, s+u) \frac{\partial}{\partial z}
\end{aligned}
$$

Thus

$$
\tau(s+u)=\omega(s+u) \circ f^{s}-\omega(s) \circ f^{s}+\omega(s)+\bar{\partial} \chi(s+u)-\left[\omega(s), \chi^{(s+u)]+O\left(|u|^{2}\right) .}\right.
$$

Let $\bar{\partial}_{j}=\partial / \partial \bar{u}_{j}$. Then applying $\bar{\partial}_{j}$ to both sides of the above equality and evaluating at $u=0$ we get $\bar{\partial}_{j} \tau=\bar{\partial} \bar{\partial}_{j} \chi-\left[\omega, \bar{\partial}_{j} \chi\right]$. On the other hand, $\delta \tau=0$, so $\delta \bar{\partial} \bar{\partial}_{j} \chi-$ $\delta\left[\omega, \bar{\partial}_{j} \chi\right]=0$, or $\bar{\partial}_{j} \chi=G \delta\left[\omega, \bar{\partial}_{j} \chi\right]$. On the other hand, $\left|\bar{\partial}_{j} \chi\right|_{k} \leq$ const. $\left|\left[\omega, \bar{\partial}_{j} \chi\right]\right|_{k-1}$, and it is very easy to see that $\left|\left[\omega, \bar{\partial}_{j} \chi\right]\right|_{k-1} \leq$ const. $|\omega(s)|_{k}\left|\bar{\partial}_{j} \chi\right|_{k}$; so $\left|\bar{\partial}_{j} \chi\right|_{k} \leq$ const. $|\omega(s)|_{k}\left|\bar{\partial}_{j} \chi\right|_{k}$. Since $\omega\left(s^{0}\right)=0$, const. $|\omega(s)|_{k}<1$ if $s^{\prime}$ is sufficiently small. This means that $\left|\bar{\partial}_{j} \chi\right|_{k}=0$, so $\bar{\partial}_{j} \chi \equiv 0$. Therefore $\bar{\partial}_{j} \equiv 0$. Q.E.D.

\section{REFERENCES}

1. L. V. Ahlfors, The complex analytic structure of the space of closed Riemann surfaces, Analytic Functions, Princeton Univ. Press, Princeton, N.J., 1960, pp. 45-66. MR 23 \#A 1798.

2. - Lectures in quasiconformal mappings, Van Nostrand Math. Studies, no. 10, Van Nostrand, Princeton, N. J., 1966. MR 34 \#336.

3. A. Andreotti and E. Vesentini, Carleman estimates for the Laplace-Beltrami equation on complex manifolds, Inst. Hautes Études Sci. Publ. Math. No. 25 (1965), 81-130. MR $30 \# 5333$.

4. L. Bers, Spaces of Riemann surfaces, Proc. Internat. Congress Math. (Edinburgh, 1958), Cambridge Univ. Press, New York, 1960, pp. 349-361. MR 23 \#A 1796. 
5. - - On moduli of Riemann surfaces, Lecture notes, Eidgenössische Technische Hochschule, Zürich, 1964.

6. K. Kodaira and D. C. Spencer, On deformations of complex analytic structures. I, II, Ann. of Math. (2) 67 (1958), 328-466. MR 22 \#3009.

7. M. Kuranishi, On the locally complete families of complex analytic structures, Ann. of Math. (2) 75 (1962), 536-577. MR 25 \#4450.

8. New proof for the existence of locally complete families of complex structures, Proc. Conference Complex Analysis (Minneapolis, 1964), Springer, Berlin, 1965, pp. 142-154. MR $31 \# 768$.

9. - Deformations of complex analytic structures on compact manifolds, Seminar on Global Analysis, Montreal, 1969 (to appear).

DEPARTMENT OF MATHEMATICS, SUNY AT STONY BROOK, STONY BROOK, NEW YORK 11790 